\title{
Melanoma in Singapore: Putting our best foot forward!
}

\author{
Haur Yueh Lee ${ }^{1,2}$ MMed (Int Med), Choon Chiat $\underline{\mathrm{Oh}}{ }^{1,2}{ }_{\text {MRCP (UK) }}$
}

Melanoma is an uncommon cancer and is disproportionately reported in Caucasians with an incidence of between 14 per 100,000 person-years in North America and 35 per 100,000 in Australia. ${ }^{1}$ In Asia, the incidence is significantly lower compared to Caucasians. A recent update from the Singapore Cancer Registry showed the incidence rate has remained stable at around 0.5 per 100,000 person-years over the past 50 years. ${ }^{2}$ Despite accounting for less than $5 \%$ of skin cancers globally, melanoma is a disease of great medical burden and accounts for a high majority of skin cancer-related morbidity and mortality. It is estimated that in 2015, there were 73,780 new cases of melanoma in the US, with corresponding 9,940 deaths. ${ }^{3}$ Over the last 20 years, the Annals of the Academy of Medicine, Singapore has charted the progress of cutaneous melanoma in Singapore over 3 cohorts of patients from 1989-1998, ${ }^{4} 1998-2008,{ }^{5}$ and 1996-2015 with the latest published in this issue. ${ }^{6}$

Melanoma in Asians is unique. Besides the variation in incidence, there are differences in the clinical presentation, mechanism and prognosis. Among the various subtypes of melanoma: superficial spreading melanoma, nodular melanoma, lentigo maligna and acral lentiginous melanoma (ALM). ALM is strikingly overrepresented, accounting for $40-50 \%$ of all cutaneous melanomas seen in Asia. ${ }^{4-7}$ This contrasts with the $2-3 \%$ in Western populations. ${ }^{8}$ On presentation, patients with ALM present with lesions that are thicker, more advanced in stage, and the 10-year survival is 10-20\% lower than other subtypes of melanoma, even after adjusting for lesional thickness. The Singapore experience has been similar. As the name suggests, ALM is found in the extremities, particularly on the foot. This is in contrast to cutaneous melanoma overall, which tends to be distributed in sun-exposed areas. This is also demonstrated in Singapore's cancer registry, where sunexposed areas accounted for only $26.7 \%$ of our melanoma cases, compared to other skin cancers $(81.3 \%$ basal cell cancer occurred on sun-exposed areas, while $61.6 \%$ squamous cell cancer occurred on sun-exposed areas). ${ }^{2}$
Ultraviolet (UV) radiation is considered the primary driver for melanoma and the daily use of sunscreens has been shown to prevent cutaneous melanomas in Caucasian populations. ${ }^{1,9,10} \mathrm{UV}$ is believed to drive mutagenesis directly through the formation of ultraviolet-signature mutations such as cytosine to tyrosine (UVB) or guanine to tyrosine (UVA), as well as indirectly via the generation of free radicals. On a molecular level, $B R A F$ gene and $R A S$ gene mutations are commonly associated with melanomas in sunexposed areas, but these mutations are uncommon in acral melanomas. Instead, these melanomas are more frequently associated with chromosomal aberrations. ${ }^{1,8}$

These unique molecular phenotypes and anatomic predilection argue against UV being a key factor in ALM carcinogenesis. Strategies that focus on minimising sun exposure, the liberal use of sunscreen, avoidance of indoor tanning-long-held as the cornerstones of melanoma prevention in the West-are unlikely to be effective in the prevention of ALM in Asia. This is further supported in a recent metaanalysis, which showed that a relationship between UV exposure and melanoma in the skin of people of colour could not be demonstrated. ${ }^{11}$

If photoprotection is inadequate, what other preventive strategies should we adopt? The data from our Singapore cohorts is instructive. Besides being older, the delay to diagnosis for local ALM patients was significantly longer at 27 months compared to 12 months in other subtypes. In addition, when the Asians were compared to resident Caucasian cases, the delays to diagnosis were 22-24 months and 7-12 months, respectively. 5,6 Possible explanations include poor public awareness of ALM, examination of the soles are not routinely performed by patients and primary care physicians, and ALM is easily misdiagnosed. More than $30 \%$ of acral lentiginous melanoma has been misdiagnosed as benign lesions and these commonly include wart, callus, tinea, onychomycosis, ulcer, foreign body reaction and haematoma. ${ }^{12}$ Education efforts should therefore be aligned to the Asian

\footnotetext{
${ }^{1}$ Department of Dermatology, Singapore General Hospital, Singapore

${ }^{2}$ Duke-NUS Medical School, Singapore

Correspondence: A/Prof Haur Yueh Lee, Department of Dermatology, Singapore General Hospital, 20 College Road, Singapore 168856.

Email: lee.haur.yueh@singhealth.com.sg
} 
phenotype. Patients need to be aware of the presentation of ALM and taught how to monitor for changing lesions on the palms and soles, as well as the nails. Opportunistic screening of these areas, particularly in the middleaged and elderly, should be interwoven into our routine clinic encounters. Any lesions of concern should be referred on for further dermatological evaluation. The use of dermoscopy (a form of portable surface microscopy of the skin that is part of a routine dermatological examination) has been shown to not only differentiate ALM from benign acral lesions, but also to identify them at an earlier, potentially curative stage. ${ }^{13,14}$

Nonetheless, several questions remain: How should we best educate and empower our patients? What are the necessary red flags that should prompt medical attention? The familiar ABCDE approach (Asymmetry, Border, Colour, Diameter, Evolution), though useful in identifying superficial spreading melanoma, has not been systematically evaluated nor validated in acral lesions. An alternative approach, CUBED (Coloured lesion, Uncertain diagnosis, Bleeding, Enlarged, Delayed healing) has been proposed to assist in decision making for onward referral and biopsy. ${ }^{14}$

Mechanistically, if UV is not a driver, what are the risk factors for ALM, and are these modifiable? Several observational studies have suggested that ALM occurs in areas of pressure or with a history of trauma. This association though intriguing would need to be validated in other populations and its biological relevance understood. ${ }^{15,16}$ Lastly, with the advent of targeted therapy and immunotherapy, the treatment landscape for advance and metastatic melanoma has been transformed. The impact of these newer therapies in the Singapore cohort will be clearer with time, although recent findings suggest that the efficacy of checkpoint inhibitors in ALM may be poorer. ${ }^{18}$

In another decade, the next cohort of our Singapore melanoma patients may be reported in the Annals again. By then, the impact of the newer therapies would be clearer; and progress would have been made if the delay to diagnosis is shortened, with patients presenting at earlier stages for curative treatments. Until then, we need to put our best foot forward and start examining our patients' foot!

\section{REFERENCES}

1. Schadendorf D, van Akkooi ACJ, Berking C, et al. Melanoma. Lancet 2018;392:971-84.

2. Oh CC, Jin AZ, Koh WP. Trends of Cutaneous Basal Cell Carcinoma, Squamous Cell Carcinoma, and Melanoma among the Chinese, Malays and Indians in Singapore from 1968-2016. JAAD International. In Press.

3. Siegel RL, Miller KD, Jemal A. Cancer Statistics, 2015. CA Cancer J Clin 2015;65:5-29.

4. Tan E, Chua SH, Lim JT, et al. Malignant melanoma seen in a tertiary dermatological centre, Singapore. Ann Acad Med Singap 2001;30:414-8.

5. Lee HY, Chay WY, Tang MBY, et al. Melanoma: differences between Asian and Caucasian patients. Ann Acad Med Singap 2012;41:17-20.

6. Yeo PM, Lim ZV, Tan WD, et al. Melanoma in Singapore: A 20-year review of disease and treatment outcomes. Ann Acad Med Singap 2021;50:456-66.

7. Bradford PT, Goldstein AM, McMaster ML, et al. Acral Lentiginous Melanoma: Incidence and Survival Patterns in the United States, 1986-2005. Arch Dermatol 2009;145:427-34.

8. Chang JWC. Acral Melanoma. Unique Disease in Asia. JAMA Dermatol 2013;149:1272-3.

9. Green AC, Williams GM, Logan V, et al. Reduced melanoma after regular sunscreen use: randomized trial follow-up. J Clin Oncol 2011;29:257-63.

10. Olsen CM, Wilson LF, Green AC, et al. How many melanomas might be prevented if more people applied sunscreen regularly. Br J Dermatol 2018;178:140-7.

11. Lopes FCPS, Sleiman MG, Sebastian K, et al. UV Exposure and the risk of cutaneous melanoma in skin of color: A Systematic Review. JAMA Dermatol 2021;157:213-9.

12. Soon SL, Solomon AR, Papadopolos D, et al. Acral lentiginous melanoma mimicking benign disease: The Emory experience. J Am Acad Dermatol 2003;48:183-8.

13. Saida T, Miyazaki A, Oguchi S, et al. Significance of dermoscopic pattern in detecting malignant melanoma on acral volar skin: Results of a multicenter study in Japan. Arch Dermatol 2004;140:1233-8.

14. Darmawan CC, Jo G, Montenegro, et al. Early Detection of Acral Melanoma: A Review of Clinical, Dermoscopic, Histopathologic and Molecular Characteristics. J Am Acad Dermatol 2019; 81:805-12.

15. Jung HJ, Kweon SS, Lee JB, et al. A clinicopathological analysis of 177 acral melanomas in Koreans: relevance of spreading pattern and physical stress. JAMA Dermatol 2013;149:1281-8.

16. Green A. McCredie M, Mackie R, et al. A case control study of melanomas of the soles and palms (Austalia and Scotland). Cancer Causes Control 1999;10:21-5.

17. Nakamura Y, Namikawa K, Yoshino K, et al. Anti-PD1 checkpoint inhibitor therapy in acral melanoma: A multicenter study of 193 Japanese patients. Ann Oncol 2020;31:1198-206. 